\title{
Predicting prediabetes in a rural community: a survey among the Karen ethnic community, Thasongyang, Thailand
}

This article was published in the following Dove Press journal:

International Journal of General Medicine

29 February 2012

Number of times this article has been viewed

\author{
Thaworn Lorga' \\ Myo Nyein Aung ${ }^{1,2}$ \\ Prissana Naunboonruang \\ Payom Thinuan' \\ Nara Praipaksin ${ }^{3}$ \\ Tida Deesakul ${ }^{3}$ \\ Utumporn Inwan ${ }^{3}$ \\ Tawatchai Yingtaweesak ${ }^{4}$ \\ Pratumpan Manokulanan' \\ Srisomporn Suangkaew' \\ Apiradee Payaprom ${ }^{4}$ \\ 'Boromarajonani College of \\ Nursing Nakhon Lampang (BCNLP), \\ Lampang, Thailand; ${ }^{2}$ Department \\ of Public Health, Graduate School \\ of Medicine, Juntendo University, \\ Tokyo, Japan; ${ }^{3}$ Baan Rekati Health \\ Station, Thasongyang, Tak, Thailand; \\ ${ }^{4}$ Thasongyang Hospital, Thasongyang, \\ Tak, Thailand
}

Background: Diabetes is a growing epidemic in both urban and rural communities worldwide. Aim: We aimed to survey fasting plasma glucose (FPG) status and awareness of diabetes in the rural Karen ethnic community. We investigated the predictors of impaired fasting plasma glucose (IFG) status, which would be easily applicable for prevention of diabetes in a rural community. Materials and methods: This was a community-based cross-sectional study conducted at Thasongyang, the most north-western district in Thailand. A total of 299 Karen ethnic rural residents were included in the study. FPG, body mass index, and waist circumference were prospectively measured. We assessed the awareness of diabetes and lifestyle-related health behavior with closed questionnaires in a rural community setting.

Results: On screening for FPG, $16.72 \%$ of the Karen ethnic residents had hyperglycemia: $3.68 \%$ in the diabetic range and $13.04 \%$ in the prediabetic range respectively. After adjustment for age, sex, and BMI, waist circumference (adjusted odds ratio [aOR] 3.5, 95\% confidence interval [CI] 1.29-9.57), and having a diabetic blood relative (aOR 4.6, CI 1.81-11.71) are significant predictors of IFG status.

Conclusion: It is necessary to promote awareness of diabetes among the Karen ethnic community. Application of simple evidence-based predictors of the prediabetic state may lead to timely and effective prevention of diabetes in rural settings.

Keywords: diabetes, prediabetes, fasting plasma glucose, Thailand, Karen ethnic community, predictors, rural health

\section{Introduction}

Diabetes is a growing global epidemic affecting 346 million people worldwide. ${ }^{1}$ The prevalence of diabetes has been persistently rising and the World Health Organization (WHO) has predicted it will double by $2030^{1,2}$ It is hitting hardest in low-and middleincome developing countries which are the location of $70 \%$ of current global diabetes cases $^{3,4}$ Thailand had more than 1.5 million diabetes cases in 2000, and this is predicted to rise to 2.7 million in $2030 .^{5}$ The silent but fast-growing disease burden is affecting not only urban, but also rural populations. There is almost no literature on the awareness of diabetes and prediabetes in rural populations such as the Karen ethnic minority in Thailand.

Impaired glucose tolerance, which is also called prediabetes, is a risk factor for cardiovascular disease. ${ }^{6}$ Affected individuals are more likely to develop type II diabetes than those with normal blood glucose levels. ${ }^{7}$ Importantly, this is the time period during which lifestyle modification interventions can be applied to prevent the onset of type II diabetes mellitus. However there is limited literature on the prediction of prediabetes.
Correspondence: Myo Nyein Aung,

Research Coordinating Unit, Boromarajonani College of Nursing Nakhon Lampang (BCNLP), 268, Parkam Road, Tambol Hauwieng, Muang District, Lampang, 52000, Thailand

Tel +66 54226254

Fax +66 54225020

Email dr.myonyeinaung@gmail.com 
It is necessary to establish reliable predictors of prediabetes in rural settings so that people at risk of type II diabetes can be identified, leading to early and effective prevention. ${ }^{8}$

In this study we aimed to survey levels of prediabetes and diabetes in a sample of the Karen ethnic population, and assess the awareness of diabetes in such a rural minority population. We aimed to determine predictors of impaired fasting glucose (IFG) status which can be used in rural community health care settings.

\section{Materials and methods Ethical approval}

The study was approved by the ethical board of Boromarajonani College of Nursing Nakhon Lampang (BCNLP), Lampang, Thailand. Verbal informed consent was obtained before the fasting plasma glucose (FPG) test and the administration of the screening questionnaires.

\section{Study site}

The study was conducted in Thasongyang district, Tak province, Thailand. It is the most north-western district in Thailand and borders Myanmar at the narrow Moei River. It is a heavily forested, mountainous area and is usually crowded with displaced persons migrating across the border. The participants were residents in the hamlets situated on the hilltops.

\section{Study population}

The study population comprised 299 Karen adults who were Thai citizens living in Thasongyang. The Karen are indigenous to the Myanmar and Thai border region. ${ }^{9}$ They are considered as hill-tribes in Thailand. A few of the Karen can speak Thai but most of the elderly Karen population cannot read Thai language. No participants had any prior diabetes mellitus. We used convenience sampling in this study.

\section{Study design}

This was a cross-sectional study. FPG levels, waist circumference, body weight, height, BMI and blood pressure were measured prospectively. Interviewer-administered questionnaires were used to survey sociodemographic factors, lifestyle-related risk factors, and to assess awareness of diabetes.

\section{Study duration}

The study ran from June 1, 2011 to September 30, 2011.

\section{FPG}

FPG measurement was conducted only on those who had fasted overnight for 8 hours before the test. The FPG level was checked by sampling venous blood. Those who did not fast overnight were excluded from testing. FPG analyses were carried out at Thasongyang Hospital laboratory.

\section{Body mass index (BMI)}

Body weight was measured, using a standard measuring scale, by the public health officers. BMI was calculated as the ratio of body weight in kilograms divided by the square of the height in meters.

\section{Waist circumference (WC)}

WC was obtained by measuring the abdominal waist circumference at umbilicus level with a measuring tape. ${ }^{10}$ Pregnant women and people with ascites were not included.

\section{Blood pressure (BP)}

Systolic and diastolic BP were recorded after two measurements made 15 minutes apart. BP was measured by community nurses using a sphygmomanometer.

\section{Questionnaires}

To assess the awareness of diabetes among the Karen ethnic community, a set of questionnaires were developed by consensus among the researchers at BCNLP, and researchers from Thasongyang. The questionnaires for assessing awareness consisted of six easily comprehensible questions written in Thai. The sets of questionnaires were delivered in the Karen language by a researcher who could speak and read both Thai and Karen language. The data were collected in the villages of Thasongyang.

\section{Definitions}

IFG or prediabetes was defined as a FPG result $\geq 100 \mathrm{mg} \%$ and $<126 \mathrm{mg}^{7}$. $^{711,12}$ The cutoff point for prediabetes (100 mg\%) was used, aiming for more sensitive detection of at-risk persons and in accordance with the existing literature in Thailand. ${ }^{7}$ According to Asian and Thai cut-off values, $\mathrm{BMI} \geq 23 \mathrm{~kg} / \mathrm{m}^{2}$ is considered as overweight, $\mathrm{BMI} \geq 25$ as preobesity and BMI $\geq 27$ as obesity. ${ }^{13,14}$ In this study we defined $\mathrm{BMI} \geq 25 \mathrm{~kg} / \mathrm{m}^{2}$ as the overweight and obese risk group for public health intervention. ${ }^{15} \mathrm{WC}>80 \mathrm{~cm}$ was considered as abnormal for females and $>90 \mathrm{~cm}$ for males according to Thai guidelines. ${ }^{7}$ Hypertension was defined as either systolic blood pressure $\geq 140 \mathrm{mmHg}$ or diastolic pressure $\geq 90 \mathrm{mmHg}^{7}$ 


\section{Statistical analysis}

Continuous variables were summarized as mean and standard deviation or median and interquartile range (IQR) based on normality of data distribution. Average values were compared by Student's $t$-test or the Wilcoxon rank-sum test based on the distribution. Chi square tests and Fischer exact tests were used for comparing the proportions. Logistic regression analysis was used to determine the risk factors for prediabetes. Univariate logistic regression analysis was applied to establish the association of each predictor with the prediabetic state. The independent predictor variables were analyzed to assess significant association with the outcome variable, ie, FPG $\geq 100 \mathrm{mg} \%$. Multivariate logistic regression analysis was strictly applied to variables with $P<0.2$ in univariate analysis and medically plausible predictor variables. StataCorp LP version 11 (StataCorp, College Station, TX) was used for data management and data analysis. Statistical significance was defined as $P<0.05$ with a $95 \%$ confidence interval.

\section{Results}

\section{Characteristics of the study population}

The study population was entirely composed of Karen ethnic adults living in Thasongyang district, Tak province, Thailand. There were 299 adult participants: 176 (58.8\%) female and 123 (41.14\%) male participants. Median age was 45 years. Minimum age was 23 years and maximum age was 82 years. Most of them had no formal education $(90.72 \%)$. Almost all of the participants were farmers $(91.32 \%)$.

\section{Clinical anthropometric markers}

The median BMI of the study population was 20.56 (IQR 18.73-22.89). There were 53 persons with a BMI above $25(17.73 \%)$. The median waist circumference was $73 \mathrm{~cm}$, but $72 \mathrm{~cm}$ among males and $74 \mathrm{~cm}$ among females (Table 2). The average BMI for females was significantly higher than for males (Table 2). In addition, a larger proportion of females had a waist circumference greater than the normal cut-off value (Table 2).

Fifteen percent of the sample population had high blood pressure $\geq 140 / 90 \mathrm{mmHg}$.

\section{FPG level of the Karen-Thai ethnic community}

FPG level was tested prospectively for all 299 participants.

The average fasting blood glucose level of the study
Table I Sociodemographic characteristics of the study sample from the Karen ethnic community

\begin{tabular}{lll}
\hline Characteristics & \multicolumn{2}{l}{ Total sample $\mathbf{n}=\mathbf{2 9 9}$} \\
\cline { 2 - 3 } & Number & Percentage \\
\hline $\begin{array}{l}\text { Age (years) } \\
\text { (median, minimum-maximum) }\end{array}$ & $45(23-82)$ & \\
$\begin{array}{l}\text { Age }>35 \text { years } \\
\text { Gender }\end{array}$ & 220 & 73.58 \\
$\quad$ Male & & \\
Female & 123 & 41.14 \\
Race & 176 & 58.86 \\
$\quad$ Karen & & \\
Religion & 299 & 100 \\
$\quad$ Buddhist & & \\
$\quad$ Christian & 270 & 93.75 \\
Education & 18 & 6.25 \\
$\quad$ No formal education & & \\
Primary & 264 & 90.72 \\
High school and higher & 20 & 6.87 \\
Occupation & 7 & 2.41 \\
$\quad$ Agriculture & & \\
Daily wage earner & 263 & 91.32 \\
Government employee & 10 & 3.47 \\
Vendor & 1 & 0.35 \\
Housewife & 3 & 1.04 \\
\hline
\end{tabular}

Table 2 Clinical anthropometric measurement, plasma glucose and blood pressure of the Karen adults in the study sample $(\mathrm{n}=299)$

\begin{tabular}{|c|c|c|c|}
\hline Average & Median (IQR) & Male & Female \\
\hline Bodyweight (kg) & $\begin{array}{l}45.47 \\
(44.25-49.75)\end{array}$ & $\begin{array}{l}51.5 \\
(46-57)\end{array}$ & $\begin{array}{l}48 \\
(43-54)\end{array}$ \\
\hline Height (cm) & $\begin{array}{l}155 \\
(150-160)\end{array}$ & $\begin{array}{l}160 \\
(155-165)\end{array}$ & $\begin{array}{l}152.5 \\
(150-160)\end{array}$ \\
\hline BMI $\left(\mathrm{kg} / \mathrm{m}^{2}\right)^{\#}$ & $\begin{array}{l}20.56 \\
(18.73-22.89)\end{array}$ & $\begin{array}{l}19.79 \\
(18.43-21.4)\end{array}$ & $\begin{array}{l}20.58^{\#} \\
(18.73-22.89)\end{array}$ \\
\hline WC & $73(68-78)$ & $72(68-76)$ & $74(68-79)$ \\
\hline FPG & $\begin{array}{l}88 \\
(8 I-95)\end{array}$ & $\begin{array}{l}88 \\
(81-95)\end{array}$ & $\begin{array}{l}81.5 \\
(87-96.5)\end{array}$ \\
\hline Systolic BP & $110(100-120)$ & $110(100-120)$ & $110(90-120)$ \\
\hline Diastolic BP & $70(60-80)$ & $70(60-80)$ & $70(60-80)$ \\
\hline Proportion & $\begin{array}{l}\text { All } \\
\text { n (\%) }\end{array}$ & $\begin{array}{l}\text { Male } \\
\text { n (\%) }\end{array}$ & $\begin{array}{l}\text { Female } \\
\text { n(\%) }\end{array}$ \\
\hline $\mathrm{BMI}<25 \mathrm{~kg} / \mathrm{m}^{2}$ & $246(82.27)$ & $105(85.37)$ & $14 \mid(80.11)$ \\
\hline $\mathrm{BMI} \geq 25 \mathrm{~kg} / \mathrm{m}^{2}$ & $53(17.73)$ & $18(14.63)$ & $35(19.87)$ \\
\hline WC above normal ${ }^{+}$ & $34(11.37)$ & $3(2.44)$ & $31(17.61)^{+}$ \\
\hline WC within normal & $265(88.63)$ & $120(97.56)$ & 145 (82.39) \\
\hline Hyperglycemia $^{+}$ & $50(16.72)$ & $13(10.57)$ & $37(21.02)^{+}$ \\
\hline Normoglycemia & $249(83.28)$ & $110(89.43)$ & 139 (78.98) \\
\hline Hypertension & $45(15.05)$ & $20(16.26)$ & $25(14.20)$ \\
\hline Normal BP & $254(84.95)$ & $103(83.74)$ & $15 \mid(85.80)$ \\
\hline
\end{tabular}

Notes: ${ }^{\sharp} P=0.00$ I by Wilcoxon rank-sum test; ${ }^{+} P<0.05$ by Fischer exact test. Abbreviations: WC, waist circumference, (normal cut off point is less than $80 \mathrm{~cm}$ for females and less than $90 \mathrm{~cm}$ for males); FPG, fasting plasma glucose; Med (IQR), median and interquartile range; BMI, body mass index; BP, blood pressure. 
population was $88 \mathrm{mg} \%$ (IQR $81-95$ ). On cross-sectional screening of FPG level, $16.72 \%$ percent of the sample population had abnormally high FPG levels. Among these, $3.68 \%$ had hyperglycemia in the diabetic range ( $>125 \mathrm{mg} \%)$ and $13.04 \%$ had hyperglycemia in the prediabetic range ( $\geq 100$ but $<125 \mathrm{mg} \%$ ), as shown in Figure 1.

\section{Awareness of diabetes among the Karen-Thai}

Diabetes awareness was assessed by using a set of questionnaires (Figure 2). Most of the participants had not heard of "diabetes mellitus." Only $11.23 \%$ had previous experience of blood glucose level tests. No-one thought they had diabetes and only $2.18 \%$ thought they were at risk of diabetes. Of the study participants, $2.91 \%$ gave a history of diabetes in blood relatives.

The smoking rate was very high in the Karen ethnic community. Current smokers comprised $70 \%$ of the study sample. Alcohol consumption is also high (51.21\%). However, most of them are physically active, as they are farmers working on the hilly farms. Only $4 \%$ of the participants were sedentary workers. Most of the participants did not perceive walking and running as sports or exercise, which led to low response rates to questionnaires on exercise activity (Table 3 ).

\section{Predictors of fasting hyperglycemia}

Female sex was a risk factor for IFG in univariate analysis (crude OR 2.3, CI 1.14-4.44).

The final model included age above 35, gender, waist circumference, BMI, hypertension and having a diabetic relative. Abnormal waist circumference (aOR 3.5, CI 1.29-9.56) and having a blood relative with diabetes (aOR 4.6, CI 1.81-11.71) were persistently significant predictors

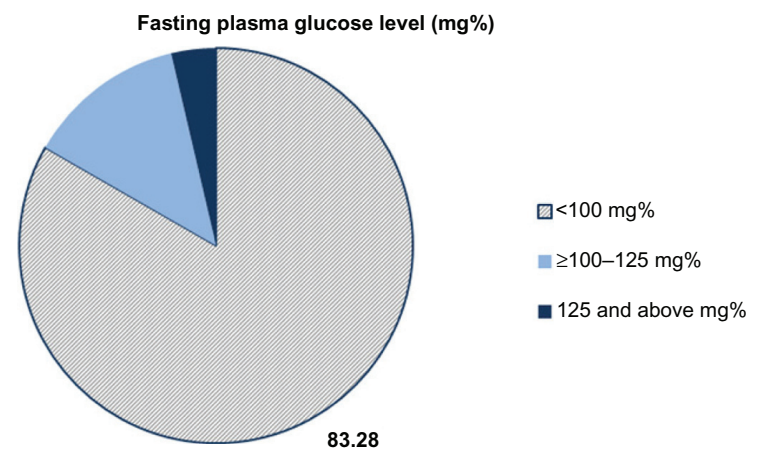

Figure I Cross-sectional screening of FPG among rural Karen ethnic adult in Thasongyang district, Thailand.

Notes: $(n=299)$, Average FPG level of the sample was $88 \mathrm{mg} \%$ (IQR 8I-95). Note $16.72 \%$ of the sample population had hyperglycemia, $13.04 \%$ with prediabetic FPG ( $\geq 100-125 \mathrm{mg} \%$ ) and $3.68 \%$ with diabetic FPG above $125 \mathrm{mg} \%$.

Abbreviations: FPG, fasting plasma glucose; IQR, interquartile range. for prediabetes after adjusting by multivariate step-wise logistic regression analysis (Table 4). Females with a waist circumference $>80 \mathrm{~cm}$ and males with a waist circumference $>90 \mathrm{~cm}$ had a significantly higher risk of having prediabetes. Individuals who had diabetic relatives were more prone to have IFG.

\section{Discussion}

The current study was conducted in a rural setting in a KarenThai community which had remarkable sociodemographic characteristics (Table 1). Most of the Karen-Thai are farmers growing hill rice. Most of them have no formal education and they use their native Karen language. Health illiteracy, communication issues, cultural barriers, financial limitations, and many other barriers present a huge challenge to health promotion in such a rural setting.

In the current study, the sample population had a median age of 45 years and $73.58 \%$ were older than 35 years. However, almost all of them ( $89 \%$ ) had never had a blood glucose test. Most had not heard of diabetes. Nobody thought themselves as diabetic (Figure 2). However, on screening for FPG levels, considerable numbers $(16.72 \%)$ of Karen people had abnormally high FPG levels and nearly 4\% were in the diabetic range (Figure 1). These indicators show that diabetes is underdiagnosed and unnoticed in this rural Karen community, with no education being provided about the disease. Similar findings of health inequity have been reported in rural communities in China. ${ }^{16}$

Most of the sample populations are farmers $(91.32 \%)$. They are usually lean, having a BMI $<25$ in $82.27 \%$ of cases (Table 1). The Karen traditional lifestyle involves working in hilly rice fields and eating their own agricultural products. However, it has increasingly changed to a more urbanized lifestyle. ${ }^{17}$ Recent research in Asia has reported that rural-to-urban migrants have a higher prevalence of obesity and diabetes than rural non-migrants. ${ }^{18}$ This is important for the public health aspect of diabetes prevention and control, as there is ongoing cross-border migration between Myanmar and Thailand.

The WHO has estimated that the number of diabetes cases worldwide will rise to 480 million by $2030 .{ }^{4}$ A current WHO noncommunicable disease report has shown the highest burden of diabetes is in low- and middle-income countries such as Thailand. ${ }^{1}$ The rural areas are not spared and potentially could be more severely hit because of poor awareness and limited health facilities. ${ }^{8}$ Moreover translating evidence to practice is difficult in rural communities. Therefore research which can be easily translated to community health care practice is urgently needed to allow intervention for the prevention of diabetes. 


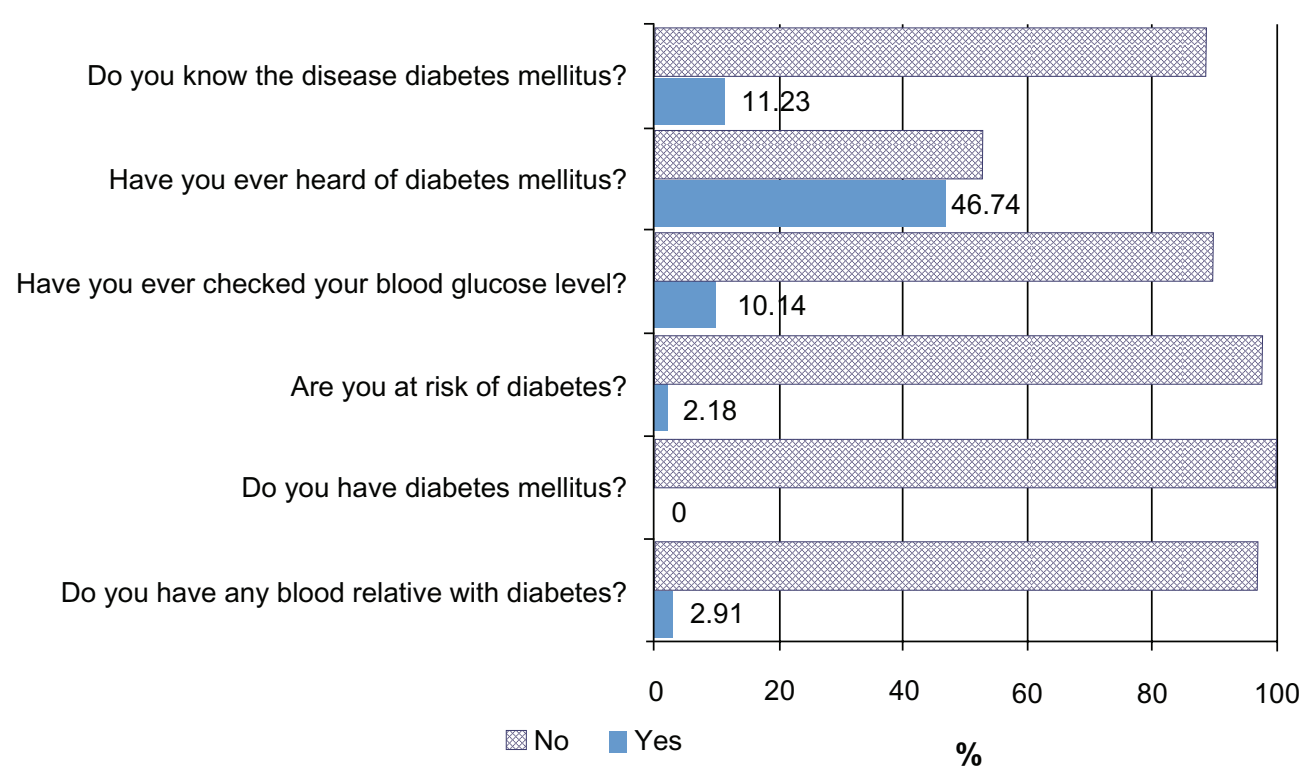

Figure 2 Awareness of diabetes mellitus amongst rural Karen ethnic adults in Thasongyang district, Thailand. $(\mathrm{n}=299), 20 \mathrm{I} \mathrm{I}$.

IFG status, or prediabetes, is a strong predictor of type II diabetes. ${ }^{6,719}$ Prediabetic persons are also at risk of cardiovascular disease and death. ${ }^{6,20}$ However, these risks can be reduced and blood glucose can be returned to normal levels by adopting a healthy lifestyle and achieving modest weight reduction during the prediabetic stage. ${ }^{20}$

In this study we used IFG status as the study outcome and analyzed easily comprehensible and rurally applicable risk markers to predict the hyperglycemic high-risk individual. After adjusting by logistic regression analysis, we identified two significant predictors of impaired fasting glucose status: abnormal waist circumference; and having a blood relative with diabetes (Table 4).

Table 3 Lifestyle-related health behaviors among rural Karen ethnic adults $(\mathrm{n}=299), 20 \mathrm{I}$

\begin{tabular}{lll}
\hline Characteristic & Number $(\mathbf{n}=\mathbf{2 9 9})$ & $\%$ \\
\hline Smoking & & \\
$\quad$ Non-smokers & 67 & 23.10 \\
Ex-smokers & 20 & 6.9 \\
$\quad$ Current smokers & 203 & 70 \\
Alcohol & & \\
Teetotaler & 103 & 35.74 \\
Ex-drinker & 38 & 13.15 \\
Current drinkers & 148 & 51.21 \\
Physical activity: exercise either walking or running until sweating & \\
5 days/week & 160 & 70.18 \\
3 days/week & 40 & 17.54 \\
Less than 3 days/week & 28 & 12.28 \\
Occupational lifestyle & & \\
Manual labor & 287 & 96 \\
Sedentary lifestyle & 11 & 4 \\
\hline
\end{tabular}

BMI and waist circumference were noted as metabolic and cardiovascular risk factors associated with mortality. ${ }^{21,22}$ Application of these indices is different between Caucasians and Asians. ${ }^{23}$ Normal body weight with increased abdominal adiposity which is called a "metabolically obese phenotype" is common in Asian populations. ${ }^{24}$ People of South East Asian descent have less muscle mass and are more prone to abdominal adiposity. Consequently, the risk of type 2 diabetes starts at a lower BMI in Asians. ${ }^{24,25}$ Central obesity indices are reported to be more associated with cardiovascular disease and diabetes than BMI in the Thai population. ${ }^{26}$ In terms of health promotion practice, waist circumference is more sensitive and appropriate than BMI in predicting individuals at high risk of prediabetes and diabetes in Asia.

Socioeconomic status of neighbors has an influence on the risk of obesity and diabetes. ${ }^{27}$ The current study, waist circumference $>80 \mathrm{~cm}$ in females and $>90 \mathrm{~cm}$ in males significantly predicted an impaired FPG state in the Karen community $(P=0.01$, Table 4$)$. Our finding is concurrent with a larger study which reported that WC predicts type 2 diabetes better than traditional cardiometabolic risk factors and BMI. ${ }^{12}$ Moreover, it is realistic and applicable in the rural setting to measure waist circumference and identify individuals at risk of prediabetes.

Socioeconomic status of neighbors has an influence on the risk of obesity and diabetes. ${ }^{27}$ In the rural Karen community, close relatives usually share a big compound and live in a cluster of houses. In the current research, we found that individuals with a positive history of diabetes among their blood relatives are at risk of 
Table 4 Predictors of impaired fasting glucose (IFG) status by univariate and multivariate logistic regression analysis

\begin{tabular}{|c|c|c|c|c|c|}
\hline Predictors of IFG status & $\begin{array}{l}\text { Crude odd ratio } \\
(95 \% \mathrm{Cl})\end{array}$ & $P$-value & $\begin{array}{l}\text { Adjusted odds ratio } \\
(95 \% \mathrm{Cl})\end{array}$ & $\begin{array}{l}\text { Prevalence } \\
(\%)\end{array}$ & $P$-value \\
\hline Age $>35$ years & $2.1(0.93-4.68)$ & 0.072 & $2.5(0.93-6.47)$ & 19.09 & Ns \\
\hline Female sex & $2.3(1.14-4.44)$ & 0.019 & $\mathrm{I} .5(0.70-3.5 \mathrm{I})$ & 21.02 & Ns \\
\hline WC $>$ normal & $3.3(1.49-7.13)$ & 0.003 & 3.5 (1.29-9.57) & 35.29 & 0.014 \\
\hline $\mathrm{BMI} \geq 25 \mathrm{~kg} / \mathrm{m}^{2}$ & $1.8(0.89-3.76)$ & 0.097 & $\mathrm{I} .08(0.4 \mathrm{I}-2.83)$ & 24.53 & Ns \\
\hline Hypertension & $1.9(0.87-4.23)$ & 0.108 & $2.15(0.91-5.07)$ & 22.22 & Ns \\
\hline Having blood relative with diabetes & $3.5(1.46-8.22)$ & 0.005 & $4.6(|.8 I-| 1.7 \mid)$ & 38.46 & 0.010 \\
\hline Smoker & $1.3(0.65-2.47)$ & 0.496 & & 17.73 & \\
\hline Alcohol drinker & $0.8(0.44-1.5 \mathrm{I})$ & 0.512 & & 15.54 & \\
\hline School education & $1.12(0.37-3.39)$ & 0.843 & & 16.29 & \\
\hline Walking until sweating 5 days/week & $0.90(0.42-1.92)$ & 0.796 & & 16.25 & \\
\hline
\end{tabular}

Abbreviations: WC, waist circumference (normal range less than $80 \mathrm{~cm}$ in females, less than $90 \mathrm{~cm}$ in males); Ns, not significant; $\mathrm{Cl}$, confidence interval; BMl, body mass index.

Note: $\mathrm{n}=299$.

impaired fasting glucose status, (aOR 4.6, CI 1.81-11.71). The risk by odds ratio was stable after adjusting for other covariables such as WC, age and BMI (Table 4). On the other hand, IFG and type II diabetes are genetically heritable, together with obesity and insulin resistance among South East Asians. ${ }^{28}$ Therefore, our finding is plausible from the point of view of a genetic as well as an environmental influence on the risk of diabetes. It could be easily used to identify prediabetic individuals in rural communities.

\section{Limitation}

In this study we have focused on predictors which would be easily applicable in community-based screening and health promotion in rural settings. Therefore anthropometric measurements and health awareness were emphasized. The dietary pattern of the Karen-Thai ethnic community might have changed to be more urban, leading to obesity and diabetes. We did not have information on the lipid profiles and dietary patterns of the participants.

\section{Recommendation}

Type II diabetes is preventable by early and effective intervention. ${ }^{1}$ Awareness of diabetes among rural and minority ethnic populations is very important for such prevention. By using simple predictors such as abnormal waist circumference, high-risk individuals can be screened for prediabetes, leading to community awareness of prediabetes and diabetes. As a result, simple life style modification measures such as reducing body weight, having regular exercise and a healthy diet, avoiding harmful use of alcohol, and cessation of smoking can be instituted earlier in the course of the disease. Thus, many cases of diabetes could be averted and the consequent cardiovascular disease burden and health economic impact would be avoided in rural communities. ${ }^{29}$

\section{Conclusion}

Impaired FPG level, or prediabetes, is a critically important time for intervention to delay or prevent the onset of type II diabetes. It must be coupled with promoting community awareness of diabetes. People have to comprehend their being at risk of diabetes to start the voluntary preventive measures.

Among apparently normal persons, abnormal waist circumference and having a blood relative with diabetes mellitus are strong predictors of prediabetes. We have pointed out easily noticeable risk factors for diabetes for the Karen ethnic and similar rural communities in Thailand and neighboring countries in Asia. It is expected that these observations are readily translatable to health promotion practice in rural communities to identify high-risk individuals and prevent the onset of diabetes.

\section{Acknowledgments}

Associate Professor Dr Hiroshi Fukuda, Department of General Medicine, Juntendo University, Tokyo, Japan is acknowledged for his important suggestions to the author for responding to the peer-reviewers. Janthila Srikrajang, Nongluk Promtingkran, Phatchanan Vivarakanon, and Suchart Kreauchai are acknowledged for their kind assistance.

\section{Disclosure}

The authors report no conflicts of interest in this work.

\section{References}

1. WHO. World Health Organization. Diabetes: Fact Sheet No 312, August 2011. Available from: http://www.who.int/mediacentre/factsheets/fs312/ en/. Accessed October 19, 2011.

2. Danaei G, Finucane MM, Lu Y, et al. National, regional, and global trends in fasting plasma glucose and diabetes prevalence since 1980: systematic analysis of health examination surveys and epidemiological studies with 370 country-years and 2.7 million participants. Lancet. 2011;378(9785):31-40. 
3. WHO. World Health Organization. Global status report on noncommunicable diseases 2010. 2011. Available from: http://www.who.int/ $\mathrm{nmh} /$ publications/ncd_report2010/en/. Accessed January 16, 2012.

4. World diabetes foundation: Diabetes facts 2011; Available from: http://www.worlddiabetesfoundation.org/composite-35.htm. Accessed October 19, 2011.

5. WHO. World Health Organization. Diabetes Programme: Country and regional data on diabetes. Available from: http://www.who.int/diabetes/ facts/world_figures/en/. Accessed October 20, 2011.

6. Levitan EB, Song Y, Ford ES, Liu S. Is nondiabetic hyperglycemia a risk factor for cardiovascular disease? A meta-analysis of prospective studies. Arch Intern Med. 2004;164(19):2147-2155.

7. Aekplakorn W, Bunnag P, Woodward M, et al. A risk score for predicting incident diabetes in the Thai population. Diabetes Care. 2006;29(8):1872-1877.

8. Massey CN, Appel SJ, Buchanan KL, Cherrington AL. Improving diabetes care in rural communities: an overview of current initiatives and a call for renewed efforts. Clin Diabetes. 2010;28(1):20-27.

9. Ethnomed [homepage on the Internet]. Neiman A, Soh E, Sutan P. Karen Cultural Profile 2008. Available from: http://ethnomed.org/ culture/karen/karen-cultural-profile. Accessed October 20, 2011.

10. Ross R, Berentzen T, Bradshaw AJ, et al. Does the relationship between waist circumference, morbidity and mortality depend on measurement protocol for waist circumference? Obesity Reviews. 2008;9(4):312-325.

11. American Diabetes Association. Diagnosis and classification of diabetes mellitus. Diabetes Care. 2004;27(Suppl 1):S5-S10.

12. Janiszewski PM, Janssen I, Ross R. Does Waist circumference predict diabetes and cardiovascular disease beyond commonly evaluated cardiometabolic risk factors? Diabetes Care. 2007;30(12):3105-3109.

13. Thaikruea L, Seetamanotch W, Seetamanotch S. Appropriate cutoff level of BMI for screening in Thai adults. J Med Assoc Thai. 2006;89(12):2123-2128

14. Low S, Chin MC, Ma S, Heng D, Deurenberg-Yap M. Rationale for redefining obesity in Asians. Ann Acad Med Singapore. 2009; 38(1):66-69.

15. WHO. World Health Organization [homepage on the Internet] Global Database on Body Mass Index. BMI classification. 2011. Available from: http://apps.who.int/bmi/index.jsp?introPage=intro_3.html. Accessed November 2, 2011.

16. Le C, Jun D, Zhankun S, Yichun L, Jie T. Socioeconomic differences in diabetes prevalence, awareness, and treatment in rural southwest China Tropi Med Int Health. 2011;16(9):1070-1076.
17. Aekplakorn W, Mo-Suwan L. Prevalence of obesity in Thailand. Obes Rev. 2009;10(6):589-592.

18. Ebrahim S, Kinra S, Bowen L, et al. The effect of rural-to-urban migration on obesity and diabetes in India: a cross-sectional study. PLoS Med. 2010;7(4):e1000268.

19. Harati H, Hadaegh F, Saadat N, Azizi F. Population-based incidence of Type 2 diabetes and its associated risk factors: results from a six-year cohort study in Iran. BMC Public Health. 2009;9:186.

20. US. Department of Health and Human Services: National Diabetes Information Clearinghouse (NDIC). Am I at risk for Type 2 Diabetes? 2008. Available from: http://diabetes.niddk.nih.gov/dm/pubs/riskfortype2/. Accessed October 21, 2011.

21. Batty GD, Shipley MJ, Jarrett RJ, Breeze E, Marmot MG, Davey Smith G. Obesity and overweight in relation to disease-specific mortality in men with and without existing coronary heart disease in London: the original Whitehall study. Heart. 2006;92(7):886-892.

22. Pischon T, Boeing H, Hoffmann K, et al. General and abdominal adiposity and risk of death in Europe. N Engl J Med. 2008;359(20):2105-2120.

23. Deurenberg P, Deurenberg-Yap M, Guricci S. Asians are different from Caucasians and from each other in their body mass index/body fat per cent relationship. Obes Rev. 2002;3(3):141-146.

24. Chan JCN, Malik V, Jia W, et al. Diabetes in Asia: epidemiology, risk factors, and pathophysiology. JAMA. 2009;301(20):2129-2140.

25. Huxley R, James WPT, Barzi F, et al. Ethnic comparisons of the crosssectional relationships between measures of body size with diabetes and hypertension. Obes Rev. 2008;9 Suppl 1:53-61.

26. Aekplakorn W, Kosulwat V, Suriyawongpaisal P. Obesity indices and cardiovascular risk factors in Thai adults. Int $J$ Obes. 2006; 30(12):1782-1790.

27. Ludwig J, Sanbonmatsu L, Gennetian L, et al. Neighborhoods, obesity, and diabetes - a randomized social experiment. $N$ Engl J Med. 2011;365(16):1509-1519.

28. Tan JT, Tan LSM, Chia KS, Chew SK, Tai ES. A family history of type 2 diabetes is associated with glucose intolerance and obesity-related traits with evidence of excess maternal transmission for obesity-related traits in a South East Asian population. Diabetes Res Clin Pract. 2008;82(2):268-275.

29. Chatterjee S, Riewpaiboon A, Piyauthakit P, et al. Cost of diabetes and its complications in Thailand: a complete picture of economic burden. Health Soc Care Community. 2011;19(3):289-298.
International Journal of General Medicine

\section{Publish your work in this journal}

The International Journal of General Medicine is an international, peer-reviewed open-access journal that focuses on general and internal medicine, pathogenesis, epidemiology, diagnosis, monitoring and treatment protocols. The journal is characterized by the rapid reporting of reviews, original research and clinical studies across all disease areas.

\section{Dovepress}

A key focus is the elucidation of disease processes and management protocols resulting in improved outcomes for the patient.The manuscript management system is completely online and includes a very quick and fair peer-review system. Visit http://www.dovepress.com/ testimonials.php to read real quotes from published authors. 